\title{
Exercise interventions for adults and children with cerebral palsy (Protocol)
}

\author{
Ryan JM, Cassidy EE, Noorduyn SG, O’Connell NE
}

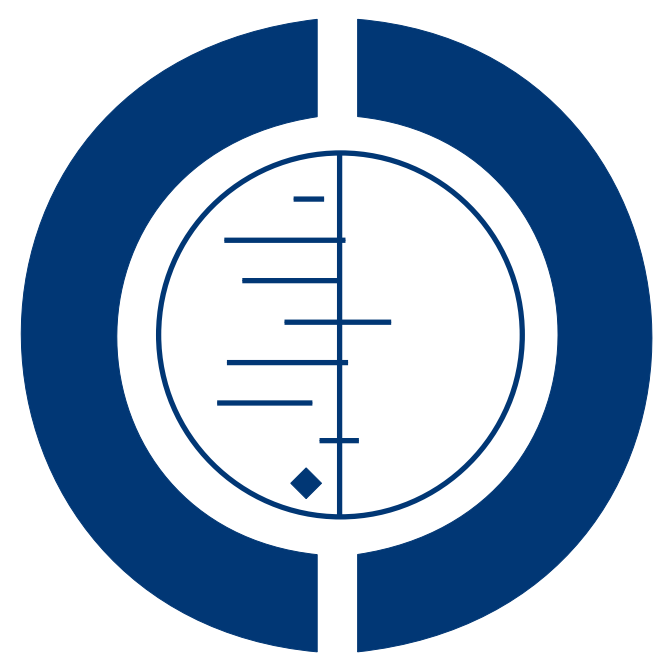

\section{THE COCHRANE COLLABORATION $^{\circledR}$}

This is a reprint of a Cochrane protocol, prepared and maintained by The Cochrane Collaboration and published in The Cochrane Library 2015, Issue 4

http://www.thecochranelibrary.com

\section{WILEY}


TABLE OF CONTENTS

HEADER . . . . . . . . . . . . . . . . . . . . . . . . . . . . . . . . . . . . 1

ABSTRACT . . . . . . . . . . . . . . . . . . . . . . . . . . . . . . . . . . . . . . . 1

BACKGROUND . . . . . . . . . . . . . . . . . . . . . . . . . . . . . . . . . . . .

OBJECTIVES . . . . . . . . . . . . . . . . . . . . . . . . . . . . . . . . . . . . . . .

METHODS . . . . . . . . . . . . . . . . . . . . . . . . . . . . . . . . . . . . . .

ACKNOWLEDGEMENTS . . . . . . . . . . . . . . . . . . . . . . . . . . . . . . . . . . . . . . . . .

REFERENCES . . . . . . . . . . . . . . . . . . . . . . . . . . . . . . . . . . . . . 8

APPENDICES . . . . . . . . . . . . . . . . . . . . . . . . . . . . . . . . . . . . . 11

CONTRIBUTIONS OF AUTHORS . . . . . . . . . . . . . . . . . . . . . . . . . . . . . . . . . . . . 16

DECLARATIONS OF INTEREST . . . . . . . . . . . . . . . . . . . . . . . . . . . . . . . 16 


\title{
[Intervention Protocol] \\ Exercise interventions for adults and children with cerebral palsy
}

\author{
Jennifer M Ryan ${ }^{1}$, Elizabeth E Cassidy ${ }^{1}$, Stephen G Noorduyn ${ }^{2}$, Neil E O’Connell $^{1}$ \\ ${ }^{1}$ Centre for Research in Rehabilitation, School of Health Sciences and Social Care, Brunel University, Uxbridge, UK. ${ }^{2}$ Department of \\ Clinical Epidemiology and Biostatistics, McMaster University, Hamilton, Canada
}

Contact address: Jennifer M Ryan, Centre for Research in Rehabilitation, School of Health Sciences and Social Care, Brunel University, Kingston Lane, Uxbridge, Middlesex, UB8 3PH, UK. jennifer.ryan@brunel.ac.uk.

Editorial group: Cochrane Developmental, Psychosocial and Learning Problems Group.

Publication status and date: New, published in Issue 4, 2015.

Citation: Ryan JM, Cassidy EE, Noorduyn SG, O'Connell NE. Exercise interventions for adults and children with cerebral palsy. Cochrane Database of Systematic Reviews 2015, Issue 4. Art. No.: CD011660. DOI: 10.1002/14651858.CD011660.

Copyright (C) 2015 The Cochrane Collaboration. Published by John Wiley \& Sons, Ltd.

\begin{abstract}
A B S T R A C T
This is the protocol for a review and there is no abstract. The objectives are as follows:

The primary aim of this systematic review is to evaluate the effect of exercise interventions on activity, participation, and health-related quality of life (HRQoL) in adults and children with CP.
\end{abstract}

The secondary aim is to evaluate the effect of exercise interventions on body functions and body structures.

\section{B A C K G R O U N D}

\section{Description of the condition}

Cerebral palsy (CP) is defined as 'a group of permanent disorders of the development of movement and posture, causing activity limitation, that are attributed to non-progressive disturbances that occurred in the developing fetal or infant brain' (Rosenbaum 2007, p 11). Children with CP may also present with cognitive impairments, hearing and visual impairments, communication difficulties, and epilepsy (Rosenbaum 2007). Most children with CP are diagnosed at around one to two years of age (Ashwal 2004; Herskind 2015) following a medical history and physical examination that identify a non-progressive motor deficit (Ashwal 2004; Rosenbaum 2007). Neuroimaging techniques, preferably magnetic resonance imaging (MRI), may be used in conjunction with the history and physical examination to establish an aetiology and prognosis (Ashwal 2004).

$\mathrm{CP}$ is the most common form of childhood disability, with prevalence rates of between 1.5 and 3.8 per 1000 live births reported in different areas of Europe and the United States (SCOPE 2002; Kirby 2011). The prevalence of CP varies not only by geographical location but also by birth weights and gestational age, with higher prevalence rates reported among children born preterm or at low birth weight (Platt 2007; Sellier 2010; Andersen 2011). Other factors associated with CP include multiple births, maternal infection during pregnancy, having a relative with $\mathrm{CP}$, breech position, and placental abruption (O'Callaghan 2011; Tollanes 2014; Tronnes 2014). The prevalence of severe CP in Europe, defined by an inability to walk and a severe intellectual disability, is approximately 0.43 per 1000 live births (SCOPE 2002). Children without severe impairments are expected to live well into adulthood (Strauss 1998a; Blair 2001). Although less is known about 
the life expectancy of adults with CP, evidence suggests that adults with CP who maintain a high level of function have a similar life expectancy to the general population (Strauss 1998b). Adults with CP therefore represent a growing population with complex healthcare needs.

The primary impairments associated with CP include reduced muscle strength (Riad 2012; Nooijen 2014), reduced cardiorespiratory fitness (Verschuren 2010; Nieuwenhuijsen 2011; Nooijen 2014), and poor selective motor control (Østensjø 2004). As a result of these impairments, children and adults with CP may have difficulty performing everyday activities such as eating, dressing, walking, running, jumping, and negotiating stairs (Østensjø 2004; Ross 2007; Opheim 2009; Klingels 2012). Intensive rehabilitation is often provided in childhood to improve gross motor function. Indeed, many children who are non-ambulatory at age two to three years will be ambulatory by the time they reach adolescence (Wu 2004). It is estimated that 54\% of five-yearold children in Europe and $56 \%$ of eight-year-old children in the United States are independently ambulatory (Beckung 2008; Kirby 2011). However, a subsequent decline in gross motor function often occurs in adolescence and young adulthood (Bottos 2001; Sandström 2004; Hanna 2009; Kerr 2011). Up to 50\% of adults with CP report experiencing deterioration in walking function from young adulthood (Bottos 2001; Opheim 2009). Many adults with CP attribute deterioration in walking function to reduced muscle strength, reduced cardiorespiratory fitness, fatigue, and pain (Jahnsen 2004; Opheim 2009). Conversely, adults who experience improvements or no change in walking function over time credit this to improvements in balance, muscle strength, and cardiorespiratory fitness (Opheim 2009). Poor gross motor function may contribute to reduced quality of life and unemployment, which is high among young adults with CP (Soyupek 2010; Verhoef 2014).

Although CP is defined by the presence of motor disorders, the clinical presentation of CP can vary considerably between individuals. The heterogeneity of $\mathrm{CP}$ makes it difficult to compare individuals at one point in time or to evaluate changes in an individual's condition over time. Traditionally, CP has been classified according to type of motor abnormality (for example spasticity, dystonia, choreoathetosis, ataxia) and anatomical distribution of CP (for example bilateral, unilateral) (Rosenbaum 2007). More recently, classification systems that allow categorisation of people with CP according to their level of functional impairment have been developed; the Gross Motor Function Classification System (GMFCS) and Manual Ability Classification System (MACS) are two such systems. The GMFCS is a five-point scale that distinguishes between levels of motor function based on functional mobility and the need for assistive technology, particularly mobility aids (Palisano 1997; Palisano 2008). A full description of the GMFCS is presented in Appendix 1.To summarise, from six years of age children in Level I of the GMFCS are able to walk indoors and outdoors without assistance and can perform gross motor skills such as running and jumping. Children in Level II can also walk indoors and outdoors without assistance but have only minimal ability to perform gross motor skills like running and jumping. Children in Level III require a mobility device to walk indoors and outdoors, and may require wheeled mobility for travelling long distances. Children in Level IV use wheeled mobility in most settings. Children in Level V are limited in their ability to maintain antigravity head and trunk postures and control arm and leg movements, and are transported in a manual wheelchair in all settings. The MACS is a five-point scale that classifies how children (four years of age or older) with CP use their hands when handling objects in daily activities (Eliasson 2006). A full description of the MACS is presented in Appendix 2. Children in Level I of the MACS handle objects easily and successfully. They may have limitations in the ease of performing tasks that require speed and accuracy. Children in Level II handle most objects but with reduced quality or speed, or both. Children in Level III have difficulty handling objects and need help to prepare or modify activities. Children in Level IV can only handle a limited selection of easily managed objects in adapted situations and require continuous support and assistance. Children in Level V do not handle objects and require total assistance. Although developed for children with CP, these systems have been used successfully to classify adults with CP (Sandström 2004).

\section{Description of the intervention}

Exercise is defined as 'physical activity that is planned, structured, repetitive, and purposive in the sense that improvement or maintenance of one or more components of physical fitness is an objective' (Caspersen 1985, p 128). The components of physical fitness that exercise may improve include muscle strength, muscle endurance, and cardiorespiratory fitness. We can categorise exercise interventions as resistance training or aerobic training based on the component of fitness the exercise programme is targeting. Resistance training involves the body's muscles working or holding against an applied force. Body weight, free weights, machine weights, and elastic bands are often used to apply force (USDHHS 2008). Aerobic training involves the body's large muscles moving in a rhythmic manner for a sustained period of time (USDHHS 2008). Walking, running, cycling, and arm ergometry are examples of aerobic exercise. Many exercise programmes target muscle strength, muscle endurance and cardiorespiratory fitness, or a combination of these components. We will refer to such programmes as 'mixed training'.

\section{How the intervention might work}

The goal of treatment for people with CP has shifted from targeting impairments of the motor system to targeting activity limitations and participation restriction, where activity is defined as a person's 
ability to execute a task and participation is defined as a person's involvement in a life situation (WHO 2001). Indeed, people with CP have identified improving restricted mobility and poor upper limb function as a primary therapeutic goal (Vargus-Adams 2011). However, it is believed that there is an association between motor impairments, activity limitation and participation restriction, so targeting one may well affect another. There is extensive evidence that impairments, particularly muscle strength, is associated with activity in children with CP (Østensjø 2004; Ross 2007; Voorman 2007; Verschuren 2009; Klingels 2012; Park 2013). Although less information is available about the association between cardiorespiratory fitness and activity, aerobic training, resistance training, and mixed training have proven efficacy on activity in older adults (Liu 2009; Gine-Garriga 2014), a population who experience similar declines in physical functioning to young adults with CP (Nusselder 2005). Improvements in activity provided by exercise may translate to improved participation in mobility-based behaviours and quality of life for people with CP (Soyupek 2010; Park 2013; Bjornson 2014). Additionally, as some adults report using exercise as a treatment for pain and find it moderately effective (Engel 2002; Hirsh 2011), exercise may improve activity, participation, and quality of life by providing pain relief. The aim of this review, to investigate the effect of exercise interventions on activity, participation and quality of life in people with $\mathrm{CP}$, reflects the goals of people with CP and clinicians treating people with $\mathrm{CP}$, and therefore is of most interest to users of this review,

While the association between physical fitness and activity suggests that improving physical fitness may improve activity, the physiological, biomechanical, and neuromuscular adaptations that may occur as a result of exercise training in people with $\mathrm{CP}$ are not understood. It is also possible that the effect of exercise on activity performance may vary according to the person's baseline level of functional ability. For example, improving muscle strength in children in GMFCS Level III, who have reduced muscle strength compared to children in GMFCS Level I (Eek 2008), may result in greater improvements in activity because of their greater potential for improvement. Conversely, improvements in muscle strength may be small in people with a greater degree of functional impairment because of their inability to exercise at an adequate intensity.

\section{Why it is important to do this review}

Although CP begins in childhood, it has a lifelong impact on the individual and the healthcare system. Identification of appropriate interventions to alleviate disability throughout the lifespan of a person with CP is urgently required. Exercise is a commonly used intervention among healthcare professionals for people with $\mathrm{CP}$, partly because the importance of exercise for improving physical functioning has been identified in other populations. This is reflected in the growing number of publications on the topic of exercise in CP.
Nine reviews have investigated the effectiveness of exercise interventions in children with CP (Dodd 2002; Taylor 2005; Anttila 2008; Mockford 2008; Rogers 2008; Verschuren 2008; Scianni 2009; Butler 2010; Novak 2013). Of these reviews, eight included articles published prior to July 2008 (Dodd 2002; Taylor 2005; Anttila 2008; Mockford 2008; Rogers 2008; Verschuren 2008; Scianni 2009; Butler 2010); one included articles published prior to December 2012 (Novak 2013). Three reviews focused solely on randomised controlled trials (RCTs) (Anttila 2008; Scianni 2009; Butler 2010); the remaining six included experimental or quasiexperimental studies. Eight reviews provided a narrative summary of the evidence (Dodd 2002; Taylor 2005; Anttila 2008; Mockford 2008; Rogers 2008; Verschuren 2008; Butler 2010; Novak 2013). Only one review conducted a meta-analysis of RCTs (Scianni 2009). However, this review looked specifically at the effectiveness of muscle strengthening, rather than all exercise interventions, in children with CP.

Two reviews have investigated the effectiveness of exercise interventions in adults with CP (Dodd 2002; Jeglinsky 2010). These reviews included observational studies published prior to March 2002 and 2009, respectively. Both reviews conducted descriptive analyses.

An up-to-date and comprehensive assessment of the evidence surrounding exercise interventions in adults and children with $\mathrm{CP}$ is required to guide consumers, health professionals, and policy makers.

\section{O B J E C T I VES}

The primary aim of this systematic review is to evaluate the effect of exercise interventions on activity, participation, and health-related quality of life (HRQoL) in adults and children with CP.

The secondary aim is to evaluate the effect of exercise interventions on body functions and body structures.

\section{METHODS}

\section{Criteria for considering studies for this review}

\section{Types of studies}

RCTs and quasi-randomised trials (where sequence generation is systematically determined but not truly random, for example based on order of entry or date of birth). 


\section{Types of participants}

Children and adults with CP of any age and functional ability.

\section{Types of interventions}

We will include studies of resistance training, aerobic training, anaerobic training, and studies that use a combination of exercises, where at least one exercise is categorised as resistance training, aerobic training, or anaerobic training (that is 'mixed training'). We will include interventions that target both the upper and lower limbs. We will not include studies of constraint-induced movement therapy, as this intervention does not specifically target one or more components of physical fitness.

Comparisons of interest are exercise versus no treatment or usual care, and comparisons of one type of exercise intervention versus another.

\section{Types of outcome measures}

\section{Primary outcomes}

1. Activity as defined as a person's ability to execute a task (WHO 2001).

Subdomains of activity are (1) activity capacity (i.e. a person's ability to execute a task in a standardised environment); (2) activity capability (i.e. a person's ability to execute a task in his or her daily environment); and (3) activity performance (i.e. what a person actually does in his or her environment) (Holsbeeke 2009).

Examples of outcome measures for activity include the Gross Motor Function Measure (GMFM), Assisted Hand Assessment (AHA), timed walk tests, Gait Profile Score (GPS), Melbourne Assessment of Unilateral Upper Limb Function (MAUULF), ABILHAND-Kids questionnaire, Activities Scale for Kids (ASK), International Physical Activity Questionnaire (IPAQ), accelerometers, and pedometers.

2. Participation as defined as a person's involvement in a life situation. This may include participation in domestic life (e.g. acquiring a place to live; managing a household; participation in employment or education; participation in community, social, and civic life) (WHO 2001).

Examples of outcome measures for participation include the Paediatric Evaluation of Disability Inventory (PEDI), Waisman Activities of Daily Living Scale (W-ADL), Functional Independence Measure (FIM), Assessment of Life Habits questionnaire (LIFE-H), and the Functional Independence Measure for Children (WeeFIM).

3. Health-Related Quality of Life (HRQoL) as defined as the impact of disease and treatment on physical, psychological, and social functioning (Schipper 1996; Solans 2008).

Examples of outcome measures for HRQoL include the Short Form- 36 (SF-36) health survey and the Child Health Questionnaire (CHQ).
4. We will also record the incidence and nature of adverse events such as injury, cardiac events, stiffness, and delayed onset muscle soreness.

\section{Secondary outcomes}

1. Body functions and body structures as defined as changes in physiological systems or in anatomical structures (WHO 2001). These include muscle strength and endurance, as measured by for example dynamometry, cardiorespiratory fitness, as measured by for example the Shuttle Run Test (SRT), pain, as measured by for example the Visual Analogue Scale (VAS), fatigue as measured by for example the Fatigue Severity Scale, and depression as measured by for example the Center for Epidemiological Studies Depression Scale (CES-D).

We will include studies that use any validated scale that measures these primary and secondary outcomes. We will collect outcomes for the following time points: short term ( 0 to less than 1 month post-intervention), intermediate term (1 month to less than 6 months post-intervention), and long term (equal to or greater than 6 months post-intervention). We will present all available results for the primary outcomes in a 'Summary of findings' table.

\section{Search methods for identification of studies}

\section{Electronic searches}

We will search the following databases for all available years.

1. Cochrane Central Register of Controlled Trials

(CENTRAL), part of The Cochrane Library

2. Ovid MEDLINE

3. Embase (OVID)

4. CINAHL (EBSCOhost)

5. Cochrane Database of Systematic Reviews (CDSR), part of The Cochrane Library

6. Database of Abstracts of Reviews of Effects (DARE), part of The Cochrane Library

7. PEDro (www.pedro.org.au/)

8. Science Citation Index (Web of Science)

9. Conference Proceedings Citation Index-Science (Web of Science)

10. UKCRN Study Portfolio (http://public.ukcrn.org.uk/ search/)

11. Health Services Research Projects in Progress (HSRPRoj) ( wwwcf.nlm.nih.gov/hsr_project/home_proj.cfm)

12. ClinicalTrials.gov (clinicaltrials.gov/)

13. World Health Organization International Clinical Trials Registry Platform (ICTRP) (www.who.int/ictrp/en/)

14. OpenGrey (www.opengrey.eu/)

15. National Rehabilitation Information Center ( www.naric.com/) 
16. LILACS (lilacs.bvsalud.org/en/)

We will use the following search strategy for Ovid MEDLINE, which incorporates the Cochrane highly sensitive search strategy for identifying randomised trials (Lefebvre 2008). We will adapt this strategy as appropriate for other sources. We will not limit searches by language, date, or publication status.

1 cerebral palsy/

2 cerebral pals\$.tw.

3 ((Hemiplegi\$ or diplegi\$ or quadriplegi\$ or unilateral\$) adj5 spastic\$).tw.

4 ((Hemiplegi\$ or diplegi\$ or quadriplegi\$ or unilateral\$) adj3 ataxi\$).tw.

5 Little\$ disease.tw.

6 or/ $1-5$

7 exp Exercise/

8 exp Exercise Movement Techniques/

9 exp Exercise Therapy/

10 exp "Physical Education and Training"/

11 Physical Endurance/

12 Physical Fitness/

13 exp Sports/

14 Hydrotherapy/

15 Equine-Assisted Therapy/

16 aerobic\$.tw.

17 (cycle or cycling).tw.

18 arm ergometry.tw.

19 treadmill.tw.

20 ergometry/

21 (exercise\$ or strength\$ or fitness).tw.

22 (flexibility or stretching).tw.

23 ((weight $\$$ adj1 lift\$) or weight training).tw.

24 (hippotherapy or equine\$ therapy or equine assist\$ or horse \$).tw.

25 sport\$.tw.

26 (walking or running).tw.

27 (aquatic\$ or swim\$).tw.

28 Physical Therapy Modalities/

29 (physiotherapy or physical therapy).tw.

30 (resistance or resisted).tw.

31 physical activit\$.tw.

32 or/7-31

33 randomized controlled trial.pt.

34 controlled clinical trial.pt.

35 randomi\#ed.ab.

36 placebo\$.ab.

37 drug therapy.fs.

38 randomly.ab.

39 trial.ab.

40 groups.ab.

41 or/33- 40

42 exp animals/ not humans.sh.

4341 not 42
446 and 32 and 43

\section{Searching other resources}

We will search reference lists of eligible trials and relevant systematic reviews that we identify with the search.

\section{Data collection and analysis}

\section{Selection of studies}

Two review authors (JR and EC) will independently check the titles and abstracts of the search results and exclude studies that do not meet the inclusion criteria outlined above. In cases that appear to meet the inclusion criteria, or where there is any doubt as to whether the article should be excluded, we will retrieve the full text of the article. Two review authors (JR and EC) will independently review these papers against the inclusion criteria. Any disagreements between review authors regarding the exclusion of an article at any stage will be resolved through discussion. If a resolution cannot be achieved, a third review author (SN) will consider the citation. Dr. Brian W. Timmons, Research Director of the Child Health \& Exercise Medicine Program and an Investigator with the CanChild Centre for Childhood Disability Research from McMaster University (Hamilton, Canada), will validate the final list of studies.

\section{Data extraction and management}

Two review authors (JR and EC) will extract data independently using a standardised form developed for the purpose. We will resolve disagreements regarding the extraction of data by discussion. If we cannot reach a resolution, we will consult a third review author (NOC). The form will include the following:

- Country of origin

- Study design

- Sample size: treatment and control groups

- Study population (treatment and control groups): sex, age, ethnicity, distribution of CP, type of motor abnormality, and gross motor function. Where sufficient information is provided, we will classify children and adults to a GMFCS level or a MACS level, or both, as it is believed that these scales provide a comprehensive indication of functional ability above that provided by classifying individuals according to type of motor abnormality and anatomical distribution of CP. In addition, we will classify general gross motor function as unaided walking, walking with aids, or unable to walk (Beckung 2008). Where information regarding GMFCS level but not general gross motor function is provided, we will collapse GMFCS levels into one of the three categories. 
- Intervention: aim of the intervention, type of exercise programme (e.g. aerobic exercise), mode of delivery (e.g. home programme), type(s) of location(s) where the intervention occurred (including any necessary infrastructure or relevant features), supervised or unsupervised programme, exercise mode (e.g. cycle ergometry, treadmill), exercise dose (i.e. duration, intensity, and frequency of exercise), tailoring of intervention to individual, modification of intervention (what, why, when, how), duration of programme

- Intervention provider: profession, expertise, background, specific training received

- Fidelity or adherence to programme: how or by whom this was assessed

- Outcome measures

- Results: short term (0 to $<1$ month post-intervention), intermediate ( 1 month to $<6$ months post-intervention), and long term ( $\geq 6$ months post-intervention) follow-up

- Measures of adherence or compliance to the exercise programme

- Adverse effects

- Conflicts of interest

- Declarations of conflicts of interest

\section{Assessment of risk of bias in included studies}

We will assess risk of bias using The Cochrane Collaboration's tool for assessing risk of bias (Higgins 2011). Two review authors (JR and EC) will independently check risk of bias. A third review author (SN) will resolve any disagreements. We will assess the following criteria: random sequence generation, allocation concealment, blinding of participants and personnel, blinding of outcome assessment, incomplete outcome data, selective reporting, and other sources of bias. We will clearly score all criteria as low risk of bias, high risk of bias, or unclear (ambiguous) risk of bias. We provide the operational definitions for making judgements on each criterion in Appendix 3.

We will assign included studies an overall rating of high, low, or unclear risk of bias. Where we rate one or more criteria at high risk of bias, we will rate that study at high risk of bias overall. Where we do not rate a study at high risk of bias for any criteria but rate it at unclear risk of bias on one or more criteria, we will rate that study at unclear risk of bias overall. We will only rate a study at low risk of bias overall if we rate it as such for all criteria.

\section{Measures of treatment effect}

\section{Binary data}

Where studies have used dichotomous outcomes (for example 'treatment response'), we will present the relative risk with $95 \%$ confidence intervals (CI) and calculate the number needed to treat for an additional beneficial outcome as an absolute measure of treatment effect where possible. Clinically important differences (CID) have been developed for a number of outcome measures (for example GMFM, weeFIM, 1-minute walk test) for ambulatory children (GMFCS Levels I to III), aged 4 to 19 years (Oeffinger 2008; Hassani 2014). However, well-established and accepted thresholds for CID have not been established across the range of possible outcome measures and range of possible participants to be included in this review. Where possible, we will consider the size of effects for our primary outcomes in light of contemporary research literature on CID in our discussion of the results.

\section{Continuous data}

Where pooled studies have used the same scale on a continuous outcome measure, we will present the effect size as mean difference with 95\% CI. Where studies have used different scales to measure the same construct, or have used different versions of an outcome measure that score the outcome differently, we will present the standardised mean difference (SMD) with 95\% CI.

\section{Unit of analysis issues}

\section{Cluster trials}

We will seek direct estimates of the effect from an analysis that accounts for the cluster design. Where the analysis in a cluster trial does not account for the cluster design, we will use the approximately correct analysis approach presented in the Cochrane Handbook for Systematic Reviews of Interventions (Higgins 2011). Using the number of clusters (or groups) randomised to each intervention group or the mean size of each cluster, the outcome data ignoring the cluster design for the total number of individuals (for example number or proportion of individuals with events, or means and standard deviations), and an estimate of the intracluster (or intraclass) correlation coefficient (ICC), we will calculate the 'effective sample size' for each cluster trial using the method described by Higgins 2011. If the ICC is not available from the study report, we will contact the authors or obtain external estimates from similar studies. If we are unable to identify an appropriate ICC, we will conduct sensitivity analyses (Sensitivity analysis) using a range of possible ICCs $(0.20,0.10,0.05,0.01)$. We will enter these data into a meta-analysis using the generic inverse-variance method (Higgins 2011).

\section{Cross-over trials}

We will enter cross-over trials into a meta-analysis when it is clear that data are free from carry-over effects. We will combine the results of cross-over studies with those of parallel studies by imputing the post-treatment between-condition correlation coefficient 
from an included study that presents individual participant data and use this to calculate the standard error of the SMD. We will enter these data into a meta-analysis using the generic inversevariance method (Higgins 2011). It is likely that included studies might present repeated measurements over time. We will only include data from one time point from an individual study in any single meta-analysis. If inadequate data are available to conduct this analysis, we will only include data from the first phase of the cross-over trial as if it were from a parallel trial design.

\section{Studies with multiple treatment groups}

Where studies include multiple treatment groups, we will combine results across all eligible intervention groups and compare them with the combined results across all eligible control groups, making single pair-wise comparisons. We will investigate possible heterogeneity through preplanned subgroup analysis (Subgroup analysis and investigation of heterogeneity).

\section{Dealing with missing data}

Where a study report presents insufficient data to enter into the meta-analysis, we will request from the authors reasons for missing data and, if available, access to missing data, with two reminder requests sent at monthly intervals in the event of nonresponse. We will specifically request data relating to the effect of the intervention on any of the outcomes of interest (for example means and standard deviations, adverse events) and details of dropouts. We will not routinely request other methodological details or information relating to the risk-of-bias assessments. We will include all studies in the main analysis and exclude studies that are at high risk of bias for incomplete outcome data as a sensitivity analysis (see Appendix 3 for risk-of-bias criteria). We will conduct analyses using only the available data. We will not impute missing data.

\section{Assessment of heterogeneity}

We will assess clinical variation across studies by comparing the distribution of important factors among trials (for example participant age, gender, and functional ability (GMFCS level), characteristics of the interventions). We will assess statistical heterogeneity and its impact using the $\mathrm{Chi}^{2}$ test and the $\mathrm{I}^{2}$ statistic (Higgins 2011). We will use the $\mathrm{Chi}^{2}$ test to determine whether differences in effects across studies are compatible with chance alone and the $\mathrm{I}^{2}$ statistic to describe the percentage of the variability in effect estimates that is due to heterogeneity rather than sampling error (chance).

\section{Assessment of reporting biases}

We will consider the possible influence of publication and smallstudy biases on review findings. Where we identify sufficient data (equal to or greater than 10 studies in a meta-analysis), we will examine funnel plots and consider the use of the test proposed by Egger 1997 to test for funnel plot asymmetry. Where we identify evidence of publication bias, we will consider its likely influence on the observed effect sizes in our interpretation of the results. However, we are aware that common tests of publication bias lack sensitivity, and so where indicated we will consider the possible influence that a dominance of small trials might have on pooled effect sizes in our interpretation.

\section{Data synthesis}

We will pool the results from included studies using Review Manager 5.3 software (Review Manager 2014). Comparisons of interest are exercise versus no treatment or usual care, and comparisons of one type of exercise intervention versus another. We will not include exercise versus no treatment or usual care, and exercise versus exercise in a single meta-analysis. We believe that the effect sizes for each of these comparisons are likely to vary considerably and that it is not theoretically justifiable to include exercise and usual care in one comparison group. Where studies compare two types of exercise interventions, we will interpret and discuss the results in the context of the evidence, or lack of evidence, of the effectiveness of each exercise intervention compared to usual care or no treatment. We will attempt to deal with clinical heterogeneity by performing separate meta-analyses for each category of exercise intervention (that is resistance training, aerobic training, anaerobic training, and mixed training). We believe that the type of exercise performed will impact the effect size and that combining these interventions will mask the true effect of each individual intervention. We will also perform separate meta-analyses for studies of adults and children. We will use a random-effects model to combine studies since we expect studies to vary somewhat in terms of the interventions, comparisons, and populations. We will consider separate meta-analyses for different types of exercise intervention and for short term ( 0 to less than 1 month post-intervention), intermediate term ( 1 month to less than 6 months postintervention), and long term (equal to or greater than 6 months post-intervention) outcomes. Where meta-analysis is not possible, we will conduct a narrative synthesis of the data. We will also consider contextual data in our interpretation of the evidence.

We will use the GRADE approach to assess the quality of the body of evidence (Guyatt 2008). To ensure consistency of GRADE judgements, we will apply the following criteria to each domain equally for all key comparisons:

- Limitations of studies: downgrade once if more than $75 \%$ of included studies are at low risk of bias across all risk-of-bias criteria.

- Inconsistency: downgrade once if heterogeneity is statistically significant ( $\mathrm{P}$ value $<0.10$ ) and $\mathrm{I}^{2}>40 \%$.

- Indirectness: downgrade once if more than $50 \%$ of the participants are outside the target group.

- Imprecision: downgrade once if fewer than 400 participants 
for continuous data and fewer than 300 events for dichotomous data (Guyatt 2011).

- Publication bias: downgrade where there is direct evidence of publication bias.

\section{Subgroup analysis and investigation of heterogeneity}

We will further explore possible clinical heterogeneity through preplanned subgroup analysis based on important clinical features. We predict that some studies will include ambulatory participants only (i.e. people who can walk with or without a mobility aid; GMFCS Level I, II, and III), and some studies will include participants who can walk without a mobility aid only (i.e. GMFCS Level I and II). Where adequate data allows, we plan to undertake two subgroup analyses for studies that include ambulatory people only (i.e. GMFCS Level I, II, and III), and for studies that include ambulatory people who walk without a mobility aid only (i.e. GMFCS Level I and II). We will interpret subgroup analyses with caution due to their exploratory nature.

\section{Sensitivity analysis}

We will explore the impact of studies at high risk of bias by reanalysis with studies rated at overall high risk of bias excluded. We will explore the impact of excluding studies at high risk of bias for missing data (see Dealing with missing data) by reanalysing with those studies included. We will assess the influence of our analysis model by reanalysing data using a fixed-effect instead of a random-effects model. We will explore the influence of using imputed correlation coefficients in our approach to including crossover and cluster trials (see Unit of analysis issues) by reanalysing these data with adjusted (higher and lower) coefficient values.

\section{ACKNOWLEDGEMENTS}

The review authors would like to thank the Cochrane Developmental, Psychosocial, and Learning Problems Group, in particular Geraldine Macdonald and Joanne Wilson.

\section{R E F E R E N C E S}

\section{Additional references}

\section{Andersen 2011}

Andersen GL, Romundstad P, De La Cruz J, Himmelmann K, Sellier E, Cans C, et al. Cerebral palsy among children born moderately preterm or at moderately low birthweight between 1980 and 1998: a European register-based study. Developmental Medicine \& Child Neurology 2011;53(10): 913-9. [PUBMED: 21838820]

\section{Anttila 2008}

Anttila H, Autti-Rämö I, Suoranta J, Mäkelä M, Malmivaara A. Effectiveness of physical therapy interventions for children with cerebral palsy: a systematic review. $B M C$ Pediatrics 2008;8:14. [DOI: 10.1186/1471-2431-8-14]

\section{Ashwal 2004}

Ashwal S, Russman BS, Blasco PA, Miller G, Sandler A, Shevell M, et al. Practice parameter: diagnostic assessment of the child with cerebral palsy: report of the Quality Standards Subcommittee of the American Academy of Neurology and the Practice Committee of the Child Neurology Society. Neurology 2004;62(6):851-63. [PUBMED: 15037681]

\section{Beckung 2008}

Beckung E, Hagberg G, Uldall P, Cans C. Probability of walking in children with cerebral palsy in Europe. Pediatrics 2008;121(1):e187-92. [PUBMED: 18070932]

Bjornson 2014

Bjornson KF, Zhou C, Stevenson RD, Christakis D. Relation of stride activity and participation in mobilitybased life habits among children with cerebral palsy. Archives of Physical Medicine and Rehabilitation 2014;95(2):360-8.
Blair 2001

Blair E, Watson L, Badawi N, Stanley FJ. Life expectancy among people with cerebral palsy in Western Australia. Developmental Medicine o Child Neurology 2001;43(8): 508-15. [DOI: 10.1111/j.1469-8749.2001.tb00753.x]

\section{Bottos 2001}

Bottos M, Feliciangeli A, Sciuto L, Gericke C, Vianello A. Functional status of adults with cerebral palsy and implications for treatment of children. Developmental Medicine \& Child Neurology 2001;43(8):516-28. [DOI: 10.1111/j.1469-8749.2001.tb00755.x]

Butler 2010

Butler JM, Scianni A, Ada L. Effect of cardiorespiratory training on aerobic fitness and carryover to activity in children with cerebral palsy: a systematic review. International Journal of Rehabilitation Research 2010;33(2): 97-103. [DOI: 10.1097/MRR.0b013e328331c555]

Caspersen 1985

Caspersen CJ, Powell KE, Christenson GM. Physical activity, exercise, and physical fitness: definitions and distinctions for health-related research. Public Health Reports 1985;100(2):126-31. [PUBMED: 3920711]

\section{Dodd 2002}

Dodd KJ, Taylor NF, Damiano DL. A systematic review of the effectiveness of strength-training programs for people with cerebral palsy. Archives of Physical Medicine and Rehabilitation 2002;83(8):1157-64. [PUBMED: 12161840]

\section{Eek 2008}

Eek MN, Beckung E. Walking ability is related to muscle strength in children with cerebral palsy. Gait \& Posture 
2008;28(3):366-71. [PUBMED: 18595712]

Egger 1997

Egger M, Smith DG. Meta-analysis: potentials and promise. BMJ 1997;315(7119):1371-4.

Eliasson 2006

Eliasson AC, Krumlinde-Sundholm L, Rösblad B, Beckung E, Arner M, Ohrvall AM, et al. The Manual Ability Classification System (MACS) for children with cerebral palsy: scale development and evidence of validity and reliability. Developmental Medicine \& Child Neurology 2006; 48(7):549-54. [PUBMED: 16780622]

\section{Engel 2002}

Engel JM, Kartin D, Jensen MP. Pain treatment in persons with cerebral palsy: frequency and helpfulness. American Journal of Physical Medicine \& Rehabilitation 2002;81(4): 291-6. [PUBMED: 11953547]

\section{Gine-Garriga 2014}

Gine-Garriga M, Roque-Figuls M, Coll-Planas L, SitjaRabert M, Salva A. Physical exercise interventions for improving performance-based measures of physical function in community-dwelling, frail older adults: a systematic review and meta-analysis. Archives of Physical Medicine and Rehabilitation 2014;95(4):753-69.e3. [PUBMED: 24291597]

\section{Guyatt 2008}

Guyatt GH, Oxman AD, Vist GE, Kunz R, Falck-Ytter Y, Alonso-Coello P, et al. GRADE: an emerging consensus on rating quality of evidence and strength of recommendations. BMJ 2008;336(7650):924-6.

\section{Guyatt 2011}

Guyatt GH, Oxman AD, Kunz R, Brozek J, Alonso-Coello P, Rind D, et al. GRADE guidelines 6: rating the quality of evidence--imprecision. Journal of Clinical Epidemiology 2011;64(12):1283-93.

\section{Hanna 2009}

Hanna SE, Rosenbaum PL, Bartlett DJ, Palisano RJ, Walter SD, Avery L, et al. Stability and decline in gross motor function among children and youth with cerebral palsy aged 2 to 21 years. Developmental Medicine of Child Neurology 2009;51(4):295-302. [PUBMED: 19391185]

\section{Hassani 2014}

Hassani S, Krzak JJ, Johnson B, Flanagan A, Gorton G 3rd, Bagley A, et al. One-Minute Walk and modified Timed Up and Go tests in children with cerebral palsy: performance and minimum clinically important differences. Developmental Medicine \& Child Neurology 2014;56(5): 482-9. [PUBMED: 24843890]

\section{Herskind 2015}

Herskind A, Greisen G, Nielsen JB. Early identification and intervention in cerebral palsy. Developmental Medicine \& Child Neurology 2015; Vol. 57, issue 1:29-36. [DOI: 10.111/dmcn.12531; PUBMED: 25041565]

\section{Higgins 2011}

Higgins JPT, Green S (editors). Cochrane Handbook for Systematic Reviews of Interventions Version 5.1.0 [updated March 2011]. The Cochrane Collaboration,

2011. Available from www.cochrane-handbook.org.

\section{Hirsh 2011}

Hirsh AT, Kratz AL, Engel JM, Jensen MP. Survey results of pain treatments in adults with cerebral palsy. American Journal of Physical Medicine \& Rehabilitation 2011;90(3): 207-16. [PUBMED: 21273894]

\section{Holsbeeke 2009}

Holsbeeke L, Ketelaar M, Schoemaker MM, Gorter JW. Capacity, capability, and performance: different constructs or three of a kind?. Archives of Physical Medicine and Rehabilitation 2009;90(5):849-55. [PUBMED: 19406307]

\section{Jahnsen 2004}

Jahnsen R, Villien L, Egeland T, Stanghelle JK, Holm I. Locomotion skills in adults with cerebral palsy. Clinical Rehabilitation 2004;18(3):309-16. [PUBMED: 15137562]

\section{Jeglinsky 2010}

Jeglinsky I, Surakka J, Carlberg EB, Autti-Rämö I. Evidence on physiotherapeutic interventions for adults with cerebral palsy is sparse. A systematic review. Clinical Rehabilitation 2010;24(9):771-88. [DOI: 10.1177/0269215510367969]

\section{Kerr 2011}

Kerr C, McDowell BC, Parkes J, Stevenson M, Cosgrove AP. Age-related changes in energy efficiency of gait, activity, and participation in children with cerebral palsy. Developmental Medicine \& Child Neurology 2011;53(1):61-7. [PUBMED: 20875041]

\section{Kirby 2011}

Kirby RS, Wingate MS, Van Naarden Braun K, Doernberg NS, Arneson CL, Benedict RE, et al. Prevalence and functioning of children with cerebral palsy in four areas of the United States in 2006: a report from the Autism and Developmental Disabilities Monitoring Network. Research in Developmental Disabilities 2011;32(2):462-9. [PUBMED: 21273041]

\section{Klingels 2012}

Klingels K, Demeyere I, Jaspers E, De Cock P, Molenaers G, Boyd R, et al. Upper limb impairments and their impact on activity measures in children with unilateral cerebral palsy. European Journal of Paediatric Neurology 2012;16(5): 475-84. [PUBMED: 22244966]

Lefebvre 2008

Lefebvre C, Manheimer E, Glanville J. Chapter 6: Searching for studies. In: Higgins JPT, Green S editor(s). Cochrane Handbook for Systematic Reviews of Interventions. Chichester: John Wiley \& Sons, 2008.

Liu 2009

Liu CJ, Latham NK. Progressive resistance strength training for improving physical function in older adults. Cochrane Database of Systematic Reviews 2009, Issue 3. [DOI: 10.1002/14651858.CD002759.pub2]

\section{Mockford 2008}

Mockford M, Caulton JM. Systematic review of progressive strength training in children and adolescents with cerebral palsy who are ambulatory. Pediatric Physical Therapy 2008; 20(4):318-33. [PUBMED: 19011522] 


\section{Nieuwenhuijsen 2011}

Nieuwenhuijsen C, Van der Slot WM, Dallmeijer AJ, Janssens PJ, Stam HJ, Roebroeck ME, et al. Physical fitness, everyday physical activity, and fatigue in ambulatory adults with bilateral spastic cerebral palsy. Scandinavian Journal of Medicine \& Science in Sports 2011;21(4):535-42. [PUBMED: 20459469]

\section{Nooijen 2014}

Nooijen C, Slaman J, Van der Slot W, Stam H, Roebroeck M, Van den Berg-Emons R. Health-related physical fitness of ambulatory adolescents and young adults with spastic cerebral palsy. Journal of Rehabilitation Medicine 2014;46 (7):642-7. [PUBMED: 24714702]

\section{Novak 2013}

Novak I, McIntyre S, Morgan C, Campbell L, Dark L, Morton N, et al. A systematic review of interventions for children with cerebral palsy: state of the evidence. Developmental Medicine \& Child Neurology 2013;55(10): 885-910. [PUBMED: 23962350]

\section{Nusselder 2005}

Nusselder WJ, Looman CW, Mackenbach JP. Nondisease factors affected trajectories of disability in a prospective study. Journal of Clinical Epidemiology 2005;58(5):484-94. [PUBMED: 15845335]

\section{O'Callaghan 2011}

O'Callaghan ME, MacLennan AH, Gibson CS, McMichael GL, Haan EA, Broadbent JL, et al. Epidemiologic associations with cerebral palsy. Obstetrics \& Gynecology 2011;118(3):576-82. [PUBMED: 21860286]

\section{Oeffinger 2008}

Oeffinger D, Bagley A, Rogers S, Gorton G, Kryscio R, Abel M, et al. Outcome tools used for ambulatory children with cerebral palsy: responsiveness and minimum clinically important differences. Developmental Medicine \& Child Neurology 2008;50(12):918-25. [PUBMED: 19046185]

\section{Opheim 2009}

Opheim A, Jahnsen R, Olsson E, Stanghelle JK. Walking function, pain, and fatigue in adults with cerebral palsy: a 7-year follow-up study. Developmental Medicine \& Child Neurology 2009;51(5):381-8. [PUBMED: 19207296]

Palisano 1997

Palisano R, Rosenbaum P, Walter S, Russell D, Wood E, Galuppi B. Development and reliability of a system to classify gross motor function in children with cerebral palsy. Developmental Medicine \& Child Neurology 1997;39(4): 214-23. [PUBMED: 9183258]

\section{Palisano 2008}

Palisano RJ, Rosenbaum P, Bartlett D, Livingston MH. Content validity of the expanded and revised Gross Motor Function Classification System. Developmental Medicine \& Child Neurology 2008;50(10):744-50. [PUBMED: 18834387]

\section{Park 2013}

Park EY, Kim WH. Structural equation modeling of motor impairment, gross motor function, and the functional outcome in children with cerebral palsy. Research in Developmental Disabilities 2013;34(5):1731-9. [PUBMED: 23500167]

\section{Platt 2007}

Platt MJ, Cans C, Johnson A, Surman G, Topp M, Torrioli MG, et al. Trends in cerebral palsy among infants of very low birthweight $(<1500 \mathrm{~g})$ or born prematurely $(<32$ weeks) in 16 European centres: a database study. Lancet 2007;369 (9555):43-50. [PUBMED: 17208641]

\section{Review Manager 2014}

The Nordic Cochrane Centre, The Cochrane Collaboration. Review Manager (RevMan). 5.3. Copenhagen: The Nordic Cochrane Centre, The Cochrane Collaboration, 2014.

\section{Riad 2012}

Riad J, Modlesky CM, Gutierrez-Farewik EM, Broström E. Are muscle volume differences related to concentric muscle work during walking in spastic hemiplegic cerebral palsy? . Clinical Orthopaedics and Related Research 2012;470(5): 1278-85. [PUBMED: 21918799]

\section{Rogers 2008}

Rogers A, Furler BL, Brinks S, Darrah J. A systematic review of the effectiveness of aerobic exercise interventions for children with cerebral palsy: an AACPDM evidence report. Developmental Medicine \& Child Neurology 2008;50(11): 808-14. [DOI: 10.1111/j.1469-8749.2008.03134.x]

\section{Rosenbaum 2007}

Rosenbaum P, Paneth N, Leviton A, Goldstein M, Bax M, Damiano D, et al. A report: the definition and classification of cerebral palsy April 2006. Developmental Medicine \& Child Neurology 2007;49(Suppl s109):8-14. [DOI: 10.1111/j.1469-8749.2007.tb12610.x]

\section{Ross 2007}

Ross SA, Engsberg JR. Relationships between spasticity, strength, gait, and the GMFM-66 in persons with spastic diplegia cerebral palsy. Archives of Physical Medicine and Rehabilitation 2007;88(9):1114-20. [PUBMED: 17826455]

\section{Sandström 2004}

Sandström K, Alinder J, Oberg B. Descriptions of functioning and health and relations to a gross motor classification in adults with cerebral palsy. Disability and Rehabilitation 2004;26(17):1023-31. [DOI: 10.1080/ 09638280410001703503]

\section{Schipper 1996}

Schipper H, Clinch JJ, Olweny CLM. Quality of life studies: definitions and conceptual issues. In: Spilker B editor(s). Quality of Life and Pharmoeconomics in Clinical Trials. Philadelphia: Lippincott-Raven, 1996.

\section{Scianni 2009}

Scianni A, Butler JM, Ada L, Teixeira-Salmela LF. Muscle strengthening is not effective in children and adolescents with cerebral palsy: a systematic review. Australian Journal of Physiotherapy 2009;55(2):81-7. [PUBMED: 19463078] 


\section{SCOPE 2002}

SCOPE. Prevalence and characteristics of children with cerebral palsy in Europe. Developmental Medicine of Child Neurology 2002;44(9):633-40. [PUBMED: 12227618]

\section{Sellier 2010}

Sellier E, Surman G, Himmelmann K, Andersen G, Colver A, Krageloh-Mann I, et al. Trends in prevalence of cerebral palsy in children born with a birthweight of 2,500 $\mathrm{g}$ or over in Europe from 1980 to 1998. European Journal of Epidemiology 2010;25(9):635-42. [PUBMED: 20532622]

\section{Solans 2008}

Solans M, Pane S, Estrada MD, Serra-Sutton V, Berra $S$, Herdman M, et al. Health-related quality of life measurement in children and adolescents: a systematic review of generic and disease-specific instruments. Value in Health 2008;11(4):742-64. [PUBMED: 18179668]

\section{Soyupek 2010}

Soyupek F, Aktepe E, Savas S, Askin A. Do the self-concept and quality of life decrease in $\mathrm{CP}$ patients? Focussing on the predictors of self-concept and quality of life. Disability and Rehabilitation 2010;32(13):1109-15. [PUBMED: 20131943]

\section{Strauss 1998a}

Strauss DJ, Shavelle RM, Anderson TW. Life expectancy of children with cerebral palsy. Pediatric Neurology 1998;18

(2):143-9. [PUBMED: 9535300]

\section{Strauss 1998b}

Strauss D, Shavelle R. Life expectancy of adults with cerebral palsy. Developmental Medicine \& Child Neurology 1998;40 (6):369-75. [DOI: 10.1111/j.1469-8749.1998.tb08211.x]

\section{Taylor 2005}

Taylor NF, Dodd KJ, Damiano DL. Progressive resistance exercise in physical therapy: a summary of systematic reviews. Physical Therapy 2005;85(11):1208-23. [PUBMED: 16253049]

Tollanes 2014

Tollanes MC, Wilcox AJ, Lie RT, Moster D. Familial risk of cerebral palsy: population based cohort study. BMJ 2014; 349:g4294. [PUBMED: 25028249]

\section{Tronnes 2014}

Tronnes H, Wilcox AJ, Lie RT, Markestad T, Moster

D. Risk of cerebral palsy in relation to pregnancy disorders and preterm birth: a national cohort study. Developmental Medicine \& Child Neurology 2014;56(8):

779-85. [PUBMED: 24621110]

\section{USDHHS 2008}

U.S. Department of Health and Human Services. Physical activity guidelines for Americans. www.health.gov/ paguidelines (accessed 31 January 2014).
Vargus-Adams 2011

Vargus-Adams JN, Martin LK. Domains of importance for parents, medical professionals and youth with cerebral palsy considering treatment outcomes. Child: Care, Health and Development 2011;37(2):276-81. [DOI: 10.1111/ j.1365-2214.2010.01121.x]

\section{Verhoef 2014}

Verhoef JA, Bramsen I, Miedema HS, Stam HJ, Roebroeck ME. Development of work participation in young adults with cerebral palsy: a longitudinal study. Journal of Rehabilitation Medicine 2014;46(7):648-55. [PUBMED: 24858956]

\section{Verschuren 2008}

Verschuren O, Ketelaar M, Takken T, Helders PJ, Gorter JW. Exercise programs for children with cerebral palsy: a systematic review of the literature. American Journal of Physical Medicine and Rehabilitation 2008;87(5):404-17. [PUBMED: 17993987]

Verschuren 2009

Verschuren O, Ketelaar M, Gorter JW, Helders PJ, Takken

T. Relation between physical fitness and gross motor capacity in children and adolescents with cerebral palsy. Developmental Medicine \& Child Neurology 2009;51(11): 866-71. [DOI: 10.1111/j.1469-8749.2009.03301.x]

\section{Verschuren 2010}

Verschuren O, Takken T. Aerobic capacity in children and adolescents with cerebral palsy. Research in Developmental Disabilities 2010;31(6):1352-7. [PUBMED: 20674266]

Voorman 2007 Voorman JM, Dallmeijer AJ, Knol DL, Lankhorst GJ, Becher JG. Prospective longitudinal study of gross motor function in children with cerebral palsy. Archives of Physical Medicine and Rehabilitation 2007;88(7):871-6. [PUBMED: 17601467]

\section{WHO 2001}

World Health Organization. International Classification of Functioning, Disability and Health. Geneva: World Health Organization, 2001.

Wu 2004

Wu YW, Day SM, Strauss DJ, Shavelle RM. Prognosis for ambulation in cerebral palsy: a population-based study. Pediatrics 2004;114(5):1264-71. [PUBMED: 15520106]

\section{Østensjø 2004}

Østensjø S, Carlberg EB, Vøllestad NK. Motor impairments in young children with cerebral palsy: relationship to gross motor function and everyday activities. Developmental Medicine \& Child Neurology 2004;46(9):580-9. [DOI: 10.1111/j.1469-8749.2004.tb01021.x]

* Indicates the major publication for the study 


\section{A P P E N D I C E S}

\section{Appendix I. Gross Motor Function Classification System}

\section{Level I: walks without restrictions; limitations in more advanced gross motor skills}

Before 2nd birthday: Infants move in and out of sitting and floor sit with both hands free to manipulate objects. Infants crawl on hands and knees, pull to stand, and take steps holding onto furniture. Infants walk between 18 months and 2 years of age without the need for any assistive mobility device.

From age 2 to 4th birthday: Children floor sit with both hands free to manipulate objects. Children perform movements in and out of floor sitting and standing without adult assistance. Children walk as the preferred method of mobility without the need for any assistive mobility device.

From age 4 to 6th birthday: Children get into and out of and sit in a chair without the need for hand support. Children move from floor and chair sitting to standing without the need for objects for support. Children walk indoors and outdoors, and climb stairs. Emerging ability to run and jump.

From age 6 to 12th birthday: Children walk at home, school, outdoors, and in the community. Children are able to walk up and down curbs without physical assistance and stairs without the use of a railing. Children perform gross motor skills, such as running and jumping, but speed, balance, and coordination are limited. Children may participate in physical activities and sports depending on personal choices and environmental factors.

From age 12: Youth walk at home, school, outdoors, and in the community. Youth are able to walk up and down curbs without physical assistance and stairs without the use of a railing. Youth perform gross motor skills, such as running and jumping, but speed, balance, and coordination are limited. Youth may participate in physical activities and sports depending on personal choices and environmental factors.

\section{Level II: walks without assistive devices; limitations walking outdoors and in the community}

Before 2nd birthday: Infants maintain floor sitting but may need to use their hands for support to maintain balance. Infants creep on their stomach or crawl on hands and knees. Infants may pull to stand and take steps holding onto furniture.

From age 2 to 4th birthday: Children floor sit but may have difficulty with balance when both hands are free to manipulate objects. Children perform movements in and out of sitting without adult assistance. Children pull to stand on a stable surface. Children crawl on hands and knees with a reciprocal pattern, cruise holding onto furniture, and walk using an assistive mobility device as preferred methods of mobility.

From age 4 to 6th birthday: Children sit in a chair with both hands free to manipulate objects. Children move from the floor to standing and from chair sitting to standing but often require a stable surface to push or pull up on with their arms. Children walk without needing any assistive mobility device indoors and for short distances on level surfaces outdoors. Children climb stairs holding onto a railing but are unable to run or jump.

From age 6 to 12th birthday: Children walk in most settings. Children may experience difficulty walking long distances and balancing on uneven terrain, on inclines, in crowded areas, in confined spaces, or when carrying objects. Children walk up and down stairs holding onto a railing or with physical assistance if there is no railing. Outdoors and in the community, children may walk with physical assistance or a hand-held mobility device or use wheeled mobility when travelling long distances. Children have at best only minimal ability to perform gross motor skills such as running and jumping. Limitations in performance of gross motor skills may necessitate adaptations to enable participation in physical activities and sports.

From age 12: Youth walk in most settings. Environmental factors (such as uneven terrain, inclines, long distances, time demands, weather, and peer acceptability) and personal preference influence mobility choices. At school or work, youth may walk using a handheld mobility device for safety. Outdoors and in the community, youth may use wheeled mobility when travelling long distances. Youth walk up and down stairs holding a railing or with physical assistance if there is no railing. Limitations in performance of gross motor skills may necessitate adaptations to enable participation in physical activities and sports.

\section{Distinctions between Levels I and II:}

Exercise interventions for adults and children with cerebral palsy (Protocol) 
Compared with children in Level I, children in Level II have limitations in the ease of performing movement transitions; walking outdoors and in the community; the need for assistive mobility devices when beginning to walk; quality of movement; and the ability to perform gross motor skills such as running and jumping.

\section{Level III: walks with assistive mobility devices; limitations walking outdoors and in the community}

Before 2nd birthday: Infants maintain floor sitting when the low back is supported. Infants roll and creep forward on their stomachs. From age 2 to 4th birthday: Children maintain floor sitting often by 'W-sitting' (sitting between flexed and internally rotated hips and knees) and may require adult assistance to assume sitting. Children creep on their stomach or crawl on hands and knees (often without reciprocal leg movements) as their primary methods of self mobility. Children may pull to stand on a stable surface and cruise short distances. Children may walk short distances indoors using an assistive mobility device and adult assistance for steering and turning. From age 4 to 6th birthday: Children sit on a regular chair but may require pelvic or trunk support to maximize hand function. Children move in and out of chair sitting using a stable surface to push on or pull up with their arms. Children walk with an assistive mobility device on level surfaces and climb stairs with adult assistance. Children are frequently transported when travelling for long distances or outdoors on uneven terrain.

From age 6 to 12th birthday: Children walk using a hand-held mobility device in most indoor settings. When seated, children may require a seat belt for pelvic alignment and balance. Sit-to-stand and floor-to-stand transfers require physical assistance of a person or support surface. When travelling long distances, children use some form of wheeled mobility. Children may walk up and down stairs holding onto a railing with supervision or physical assistance. Limitations in walking may necessitate adaptations to enable participation in physical activities and sports, including a self propelling manual wheelchair or powered mobility.

From age 12: Youth are capable of walking using a hand-held mobility device. In comparison with individuals in other levels, youth in Level III demonstrate more variability in methods of mobility depending on physical ability and environmental and personal factors. When seated, youth may require a seat belt for pelvic alignment and balance. Sit-to-stand and floor-to-stand transfers require physical assistance from a person or support surface. At school, youth may self propel a manual wheelchair or use powered mobility. Outdoors and in the community, youth are transported in a wheelchair or use powered mobility. Youth may walk up and down stairs holding onto a railing with supervision or physical assistance. Limitations in walking may necessitate adaptations to enable participation in physical activities and sports, including self propelling a manual wheelchair or powered mobility.

\section{Distinctions between Levels II and III:}

Differences are seen in the degree of achievement of functional mobility. Children in Level III need assistive mobility devices and frequently orthoses to walk, while children in Level II do not require assistive mobility devices after age 4.

\section{Level IV: self mobility with limitations; children are transported or use power mobility outdoors and in the community}

Before 2nd birthday: Infants have head control but require trunk support for floor sitting. Infants can roll to supine and may roll to prone.

From age 2 to 4th birthday: Children floor sit when placed but are unable to maintain alignment and balance without using their hands for support. Children frequently require adaptive equipment for sitting and standing. Children achieve self mobility for short distances (within a room) through rolling, creeping on stomach, or crawling on hands and knees without reciprocal leg movement. From age 4 to 6th birthday: Children sit on a chair but need adaptive seating for trunk control and to maximize hand function. Children move in and out of chair sitting with assistance from an adult or a stable surface to push or pull up on with their arms. Children may at best walk short distances with a walker and adult supervision but have difficulty turning and maintaining balance on uneven surfaces. Children are transported in the community. Children may achieve self mobility using a power wheelchair.

From age 6 to 12th birthday: Children use methods of mobility that require physical assistance or powered mobility in most settings. Children require adaptive seating for trunk and pelvic control and physical assistance for most transfers. At home, children use floor mobility (roll, creep, or crawl), walk short distances with physical assistance, or use powered mobility. When positioned, children may use a body support walker at home or school. At school, outdoors, and in the community, children are transported in a manual wheelchair or use powered mobility. Limitations in mobility necessitate adaptations to enable participation in physical activities and sports, including physical assistance or powered mobility, or both.

From age 12: Youth use wheeled mobility in most settings. Youth require adaptive seating for pelvic and trunk control. Youth require physical assistance from one or two persons for transfers. Youth may support weight with their legs to assist with standing transfers. Indoors, youth may walk short distances with physical assistance, use wheeled mobility, or, when positioned, use a body support walker.

Exercise interventions for adults and children with cerebral palsy (Protocol)

Copyright @ 2015 The Cochrane Collaboration. Published by John Wiley \& Sons, Ltd. 
Youth are physically capable of operating a powered wheelchair. When a powered wheelchair is not feasible or available, youth are transported in a manual wheelchair. Limitations in mobility necessitate adaptations to enable participation in physical activities and sports, including physical assistance or powered mobility, or both.

\section{Distinctions between Levels III and IV:}

Differences in sitting ability and mobility exist, even allowing for extensive use of assistive technology. Children in Level III sit independently, have independent floor mobility, and walk with assistive mobility devices. Children in Level IV function in sitting (usually supported), but independent mobility is very limited. Children in Level IV are more likely to be transported or to use power mobility.

\section{Level V: self-mobility is severely limited even with the use of assistive technology}

Before 2nd birthday: Physical impairments limit voluntary control of movement. Infants are unable to maintain antigravity head and trunk postures in prone and sitting. Infants require adult assistance to roll.

From age 2 to 12th birthday: Children are transported in a manual wheelchair in all settings. Children are limited in their ability to maintain antigravity head and trunk postures and control arm and leg movements. Assistive technology is used to improve head alignment, seating, standing, and/or mobility, but limitations are not fully compensated for by equipment. Transfers require complete physical assistance of an adult. At home, children may move short distances on the floor or may be carried by an adult. Children may achieve self mobility using powered mobility with extensive adaptations for seating and control access. Limitations in mobility necessitate adaptations to enable participation in physical activities and sports, including physical assistance and using powered mobility. From age 12: Youth are transported in a manual wheelchair in all settings. Youth are limited in their ability to maintain antigravity head and trunk postures and control arm and leg movements. Assistive technology is used to improve head alignment, seating, standing, and mobility, but limitations are not fully compensated for by equipment. Transfers require physical assistance from one or two persons or a mechanical lift. Youth may achieve self mobility using powered mobility with extensive adaptations for seating and control access. Limitations in mobility necessitate adaptations to enable participation in physical activities and sports, including physical assistance and using powered mobility.

\section{Distinctions between Levels IV and V:}

Children in Level V lack independence even in basic antigravity postural control. Child achieves self mobility only if he or she can learn how to operate an electrically powered wheelchair.

\section{Appendix 2. Manual Ability Classification System}

Level I: handles objects easily and successfully. At most, limited in the ease of performing manual tasks requiring speed and accuracy. However, any limitations in manual abilities do not restrict independence in daily activities.

Level II: handles most objects but with somewhat reduced quality or speed of achievement, or both. May avoid or achieve with some difficulty certain activities; might use alternative ways of performance, but manual abilities do not usually restrict independence in daily activities.

Level III: handles objects with difficulty; needs help to prepare or modify activities, or both. The performance is slow and achieved with limited success regarding quality and quantity. Performs activities independently if they have been set up or adapted.

Level IV: handles a limited selection of easily managed objects in adapted situations. Performs parts of activities with effort and with limited success. Requires continuous support and assistance or adapted equipment, or both, for even partial achievement of the activity. Level V: does not handle objects and has severely limited ability to perform even simple actions. Requires total assistance.

Distinctions between Levels I and II. Children in Level I may have limitations in handling very small, heavy, or fragile objects, which demand detailed fine motor control or efficient coordination between hands. Limitations may also involve performance in new and unfamiliar situations. Children in Level II perform almost the same activities as children in Level I, but the quality of performance is decreased or the performance is slower. Functional differences between hands can limit effectiveness of performance. Children in Level II commonly try to simplify handling of objects, for example by using a surface for support instead of handling objects with both hands.

Distinctions between Levels II and III. Children in Level II handle most objects, although slowly or with reduced quality of performance. Children in Level III commonly need help to prepare the activity or require that adjustments be made to the environment, 
or both, since their ability to reach or handle objects is limited. They cannot perform certain activities and their degree of independence is related to the supportiveness of the environmental context.

Distinctions between Levels III and IV. Children in Level III can perform select activities if the situation is prearranged and if they receive supervision and plenty of time. Children in Level IV need continuous help during the activity and can at best participate meaningfully in only parts of an activity.

Distinctions between Levels IV and V. Children in Level IV perform part of an activity with continuous help. Children in Level V might at best participate with a simple movement in special situations, for example by pushing a button.

\section{Appendix 3. Risk of bias criteria: operational definitions}

\section{Adequate sequence generation?}

Low: based on a random component judged to be both appropriate and sufficiently well described.

High: based on any non-random component.

Unclear: insufficient information regarding sequence generation process to permit a judgement of low or high risk of bias.

\section{Adequate allocation concealment?}

Low: method of concealment allocation employed prohibited foresight of patient assignment.

High: method of concealment allocation employed permitted possible foresight of patient assignment.

Unclear: method of concealment allocation not described or described in insufficient detail to permit a judgement of low or high risk of bias.

\section{Blinding of outcome assessment?}

Low: outcome assessor (including 'patients' with respect to self report outcomes) blinded to patients' allocated intervention, and unlikely that blinding broken; OR no or incomplete blinding but judged that a given outcome unlikely to be influenced by lack of blinding. High: outcome assessor (including 'patients' with respect to self report outcomes) unblinded to patients' allocated intervention; OR outcome assessor blinded to allocated intervention but likely that blinding may have been broken (and a given outcome is likely to be influenced by lack of blinding).

Unclear: insufficient information to permit judgement of low or high risk of bias.

\section{Blinding of patients?}

Low: patients blinded to allocated intervention and unlikely that blinding broken; OR no or incomplete blinding but judged that a given outcome unlikely to be influenced by lack of blinding.

High: patients not blinded to allocated intervention; OR patients blinded to allocated intervention but likely that blinding may have been broken (and a given outcome is likely to be influenced by lack of blinding).

Unclear: insufficient information to permit judgement of low or high risk of bias.

\section{Blinding of care provider?}

Low: care provider blinded to allocated intervention and unlikely that blinding broken; OR no or incomplete blinding but judged that a given outcome unlikely to be influenced by lack of blinding.

High: care provider not blinded to allocated intervention; OR patients blinded to allocated intervention but likely that blinding may have been broken (and a given outcome is likely to be influenced by lack of blinding).

Unclear: insufficient information to permit judgement of low or high risk of bias. 


\section{Incomplete outcome data addressed?}

Low: no missing outcome data. Reasons for missing data unlikely to be related to the true outcome. Missing outcome data balanced across intervention groups with similar reasons for omissions. Dichotomous outcomes: proportion of missing outcomes compared with observed event risk not enough to have a clinically relevant impact on the intervention effect estimate. Continuous outcomes: difference in means or standardised mean difference among missing outcomes not enough to have a clinically relevant impact on observed effect size. Missing data imputed using appropriate methods. Intention-to-treat analysis undertaken. Less than or equal to $10 \%$ dropout rate. High: reason for missing outcome data likely to be related to the true outcome. Dichotomous outcomes: proportion of missing outcomes compared with observed event risk enough to induce a clinically relevant bias in intervention effect estimate. Continuous outcomes: difference in means or standardised mean difference among missing outcomes enough to induce a clinically relevant bias on observed effect size. As-treated analysis undertaken with substantial departure of the intervention received from that assigned at randomisation. Equal to or greater than $30 \%$ dropout rate.

Unclear: insufficient reporting of attrition or exclusions to permit judgement of low or high risk of bias. Greater than $10 \%$ and less than $30 \%$ dropout rate.

\section{Selective outcome reporting?}

Low: all primary outcomes of interest adequately reported with point estimates and measures of variance for all time points.

High: incomplete reporting of prespecified outcomes. One or more primary outcomes is reported using measurements, analysis methods, or subsets of data that were not prespecified. One or more reported primary outcomes were not prespecified. One or more outcomes of interest reported incompletely and cannot be entered into a meta-analysis. Results for a key outcome expected to have been reported, excluded.

Unclear: insufficient information to permit judgement of low or high risk of bias.

\section{Free of other biases}

Low: appears free of other sources of bias.

High: results may have been confounded by at least one important risk of bias (design-specific, fraudulent, other).

Unclear: other sources of bias may be present but there is either insufficient information to assess whether an important risk of bias exists; OR insufficient rationale or evidence regarding whether an identified problem will introduce bias.

\section{CONTRIBUTIONSOFAUTHORS}

JMR: Conceived and designed the review protocol; will implement the search strategy, apply eligibility criteria, assess studies, extract and analyse data; and will lead the review write-up and updating.

EEC: Informed the protocol design; will apply eligibility criteria, assess studies, extract data; and will assist with the review write-up and updating.

SGN: Informed the protocol design; will act as the third reviewer; and will assist with the review write-up and updating.

NEOC: Informed the protocol design; will oversee the data synthesis; and will assist with the review write-up and updating. 


\section{DECLARATIONSOF INTEREST}

Jennifer M Ryan, Elizabeth E Cassidy, and Neil E O'Connell are chartered physiotherapists and lecturers in physiotherapy. As professionals who might be involved in the delivery of exercise interventions, it is plausible that they might be perceived as having a bias favouring the effectiveness of exercise.

Jennifer M Ryan is receiving funding from Action Medical Research and the Chartered Society of Physiotherapy Charitable Trust to evaluate the feasibility, acceptability, and efficacy of resistance training in adolescents with cerebral palsy.

Elizabeth E Cassidy: none known.

Stephen G Noorduyn: none known.

Neil E O’Connell: none known. 